\title{
First glance at the tracking detectors data collected in the first BM@N SRC run
}

\author{
Sergey Khabarov ${ }^{1}$, Elena Kulish ${ }^{1}$, Vasilisa Lenivenko ${ }^{1, *}$, Alexander Makankin ${ }^{1}$, Anna \\ Maksymchuk $^{1}$, Vladimir Palichik ${ }^{1}$, Maria Patsyuk ${ }^{2}$, Sergey Vasiliev ${ }^{1}$, Aleksander \\ Vishnevskij ${ }^{1}$, and Nikolay Voytishin ${ }^{1}$ \\ ${ }^{1}$ Joint Institute for Nuclear Research, Joliot Curie 6, 141980 Dubna, Moscow region, Russia \\ ${ }^{2}$ Massachusetts Institute of Technology, Massachusetts Ave 77, 02139 MA, Cambridge, USA
}

\begin{abstract}
BM@N (Baryonic Matter at Nuclotron) is the first experiment at the accelerator complex of NICA-Nuclotron at JINR (Dubna). The aim of the experiment is to study collisions of relativistic ion beams of the kinetic energy from 1 to $4.5 \mathrm{AGeV}$ with fixed targets. The last run started a new physics program of BM@N - Short Range Correlations (SRC) studies in light nuclei. The BM@N setup allows detecting of the residual nucleus for the first time. $\mathrm{BM} @ \mathrm{~N}$ tracking detectors play a key role in the identification of the nucleus after hard scattering in inverse kinematics. We present the first results of the BM@N tracking detectors using the data collected in spring 2018.
\end{abstract}

\section{Introduction}

The fixed target experiment BM@N (Baryonic Matter at Nuclotron) is a part of the NICANuclotron-M which is currently under construction [1]. BM@N energies lie in the region of the production of the main strange hypernuclei and are perfectly suitable for this investigation [2].

The last BM@N data taking period took place in spring 2018. That run started the new physics program on SRC studies [3]. Short Range Correlations occur when two nucleons inside a nucleus happen to be at a close proximity. The SRC pair is characterized by high absolute momentum of the nucleons and low center of mass momentum (relative to Fermi momentum). The BM@N setup at the Nuclotron is a unique place for SRC studies as it allows performing of the residual nucleus for the first time. The BM@N experiment uses ion beams (12C in the 2018 SRC run), and the nucleus after interaction continues moving forward passing through detection systems, therefore measuring properties of the nucleus. The identification of the recoil system includes determination of its atomic weight $\mathrm{A}$ and charge Z. Dedicated counters were used to measure Z. The Multiwire Proportional Chambers (MWPC) and Drift Chambers (DCH) detector will measure the turning angles of recoil fragments, which in combination with the Time-of-Flight systems (ToF700) allows to identify the residual nuclei (A-2).

The tracking detectors of the experiment include: MWPC, Silicon Tracker, Gas Electron Multipliers, Time-of-Flight systems, Cathode Strip Chambers (CSC), DCH, neutron detector

\footnotetext{
*e-mail: vasilisa@jinr.ru
} 
(LAND), Zero Degree Calorimeter. The scheme of the SRC at BM@N experimental setup is shown in Figure 1. For this study we consider MWPC, CSC and DCH tracking detectors.

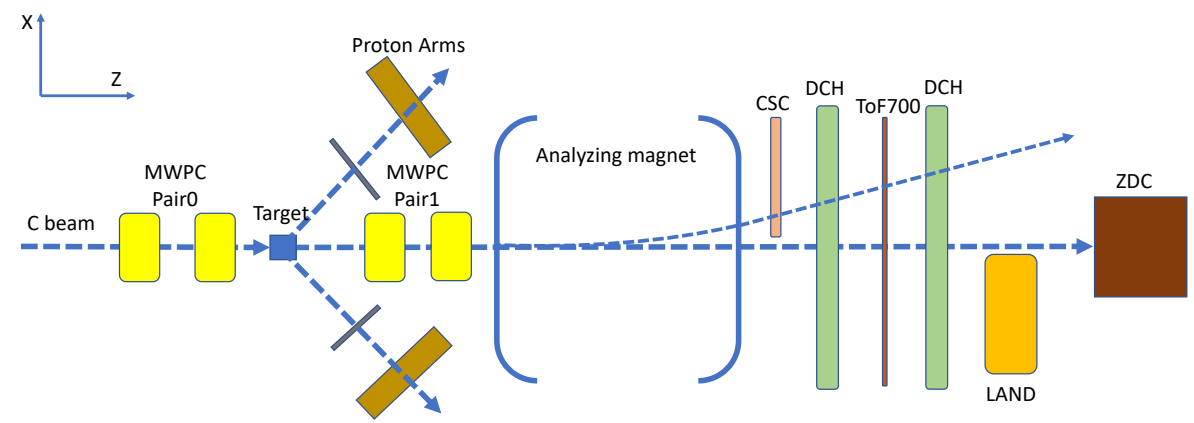

Figure 1. Schematic view of the SRC at BM@N experimental setup.

The following configuration of MWPC chambers was used in the SRC at BM@N data taking period: two MWPCs (Pair0) were located on the beamline in front of the target to measure the beam trajectory and two MWPCs (Pair1) were installed downstream the target to measure recoil products. MWPC [4] play a key role in the estimation of the momentum and identification of the residual nucleus.

\section{Reconstructed Track Parameters using MWPCs}

Each MWPC station has six coordinate planes: two X, two U and two V - planes inclined at the angles of $0^{\circ}, \pm 60^{\circ}$ to the vertical axis. The intersection of these planes defines the working area of the chamber. The coordinate resolution is $d / \sqrt{12}=0.72 \mathrm{~mm}$ for the wire pitch d of $2.5 \mathrm{~mm}$.

In previous BM@N data taking beams up to deuteron were used, so only two or three MPWC wires were fired and there was no problem with the coordinate calculation in each plane [5]. There were three variants of particle passing through the wires: between the first and second, between the second and third or through the middle wire. The spatial point was constructed from three fired wires of $\mathrm{X}, \mathrm{U}$ and $\mathrm{V}$ coordinate planes. This point should satisfy the following condition:

$$
V+U-X=0
$$

Then the algorithm looks for its conjugate point in the region no more than two pitches in next three planes of this chamber. Thereby, points for tracks candidates or segments were selected. Segments with six points were collected first, then with five and with four points. The best segment was selected by the $\chi^{2}$ criterion. Segments having common points with this best segment were rejected. Each segment was extrapolated to the middle plane between two chambers, the best matching between segments was selected by the $\chi^{2}$ criterion. The best pair of segments was fitted by the straight line, obtained parameters were the beam parameters.

The SRC at BM@N experiment used the carbon beam. The number of fired wires was higher than three (in average is six fired wires), this leads to the clustering implementation into the tracks searching algorithm. The algorithm takes into account the cluster width at the segments parameters calculation $(\sigma=$ cluster width $/ \sqrt{1} 2)$. Since there can be only one track behind the target and several tracks after it, the possibility to choose the search accuracy was 
implement by varying the number of segments and best tracks. Besides, the algorithm was improved to adopt four chambers at the same time. The method is executed sequentially for the Pair0 and then for the Pair1. Algorithm calculation time was drastically reduced. The reconstructed spot of the carbon ion beam in the target area is presented in Figure 2. The spot size is consistent with the expectations and the online monitoring results. The algorithm can be evaluated by the tracks extrapolation to the target area and the calculation of the difference between tracks from Pair0 and Pair1 in Figure 3. Sigma of this difference are $0.4883 \mathrm{~cm}$ for the $\mathrm{X}$ coordinate and $0.4788 \mathrm{~cm}$ for the $\mathrm{Y}$ coordinate. The resulting sigma are consistent with estimates based on the magnitude of the pitch and the distance between the chambers.

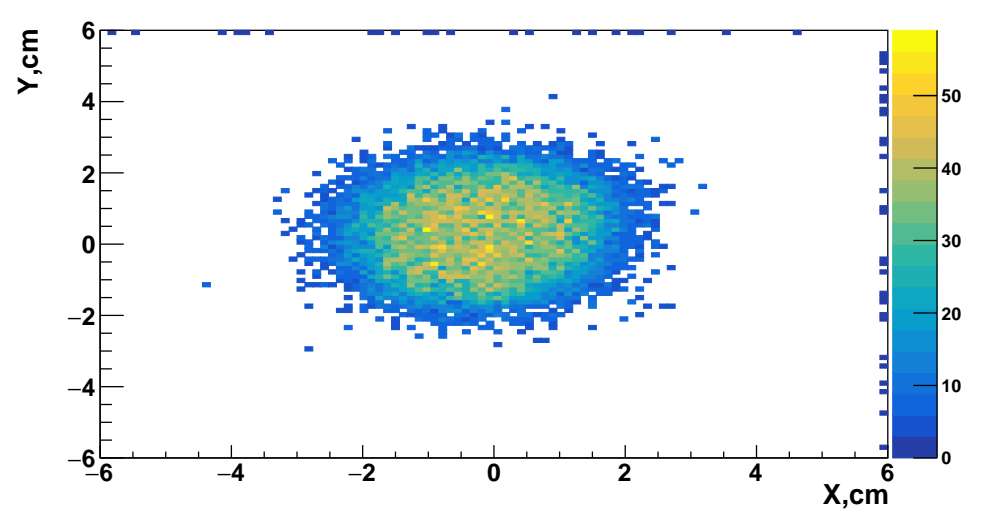

Figure 2. Reconstructed spot of the carbon ion beam in the target position, empty target.
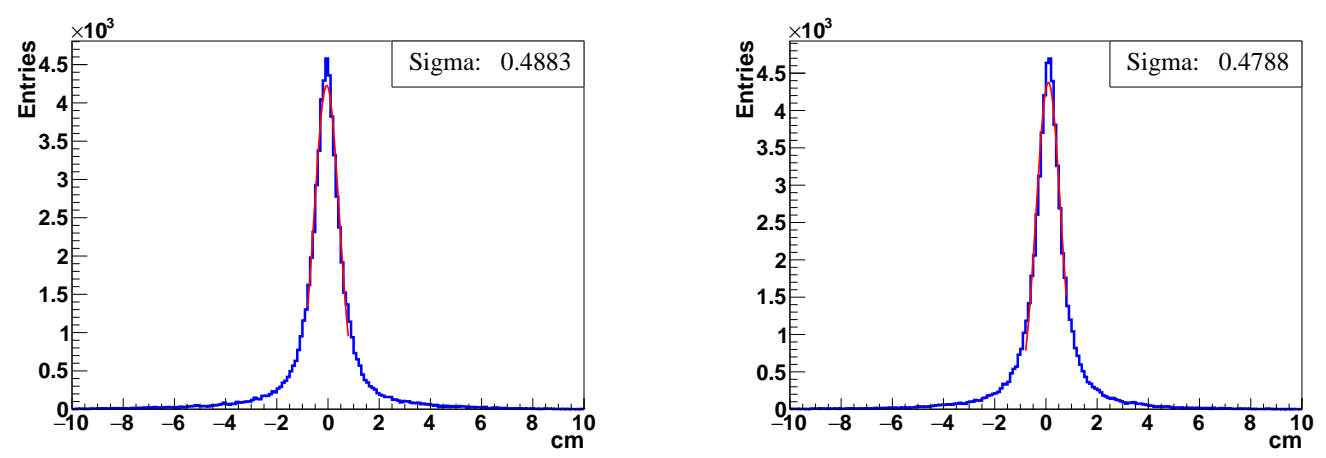

Figure 3. The difference between the track coordinates from different pairs in the target area for the $\mathrm{X}$ coordinate (left) and for the $\mathrm{Y}$ coordinate (right). Sigma are 0.4883 and 0.4788 respectively.

\section{CSC Prototype Matching to DCH}

Recoil fragments and residual nuclei deviate inside the magnetic field. The track reconstruction after the analyzing magnet for the turning angle measurement is an important task.

Two DCH [4] were installed downstream the analyzing magnet. They are used for reconstruction of the charged particles trajectory after the analyzing magnet and for linking tracks with ToF700 system for particle identification. Each DCH consists of four double coordinate 
planes with wires oriented at the angles of $0^{\circ}, 90^{\circ}$ and $\pm 45^{\circ}$. The wire pitch is $10 \mathrm{~mm}$. The best spatial resolution with the experimental data is $180 \mu \mathrm{m}$ while the average resolution is about $300 \mu \mathrm{m}$. The average reconstruction efficiency for all planes is around $90 \%$.

The CSC will allow the BM@N experiment to work with much higher luminosities than now. This detector does not depend on the starting time and the delimitation of two or more overlapped signals is possible with a very high precision. In the future DCH will be replaced by CSC. First tests of the CSC chamber prototype were performed at the Nuclotron beam during the spring 2018. The CSC [4] is installed between the analyzing magnet and the DCH chambers to improve the accuracy of track and momentum reconstruction. The active area of CSC is $107.6^{*} 112.9 \mathrm{~cm}^{2}$. The two-dimensional strip readout is divided into four zones for better capability to resolve multiple tracks. The inclination angles of $X$ and $X^{\prime}$ strips to the vertical axis are 0 and 15 degrees, respectively. The strip pitch is $2.5 \mathrm{~mm}$ for both layers. The total number of $X$ and $X^{\prime}$ strips for all four zones is 1808 and 1928, respectively. Both the design and assembly of CSC were performed at Veksler and Baldin Laboratory of High Energies Physics JINR.

Every cathode readout zone is formed from cathode strips where the charge from ion avalanches is collected to form clusters of fired strips. The average width of the cluster is six strips. The reconstruction efficiency for all zones is about $98 \%$. The correlation of DCH segments and CSC spatial hits was studied. For this purpose, tracks reconstructed in DCH were extrapolated to the CSC plane. The difference between the coordinate of the extrapolated track from DCH and the coordinate of the CSC cluster is shown in Figure 4. Sigma of this difference are $0.5589 \mathrm{~cm}$ for the $\mathrm{X}$ coordinate and $0.4377 \mathrm{~cm}$ for the $\mathrm{Y}$ coordinate.
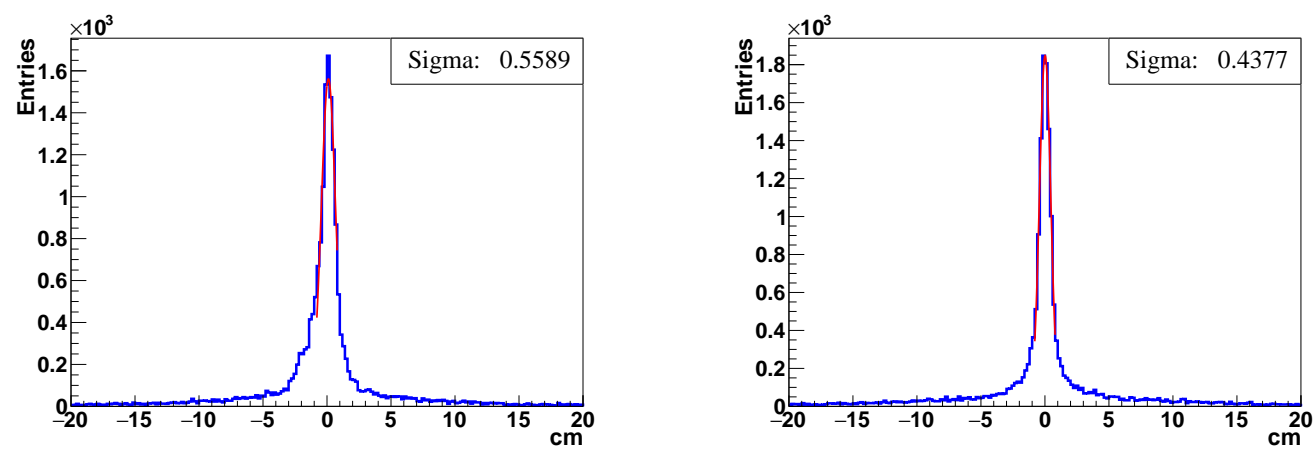

Figure 4. The difference between the coordinate of the extrapolated track from DCH and the coordinate measured in the CSC for the X coordinate (left) and for the Y coordinate (right). Sigma are 0.5589 and 0.4377 respectively.

The CSC hit reconstruction was performed for the first time at the BM@N experiment and in the future it will be added to the software package of the experiment.

\section{Conclusions}

The algorithm for track reconstruction in the MWPC was developed for the SRC program. This algorithm was significantly improved with respect to the existing one in terms of speed and adopted for the carbon beam data. Also, the described method was implemented into the general reconstruction chain of the official software package of the BM@N experiment.

The algorithm was applied to the last run SRC data, and the beam parameters in the target area were obtained. The next step is to use the MWPC reconstruction algorithm for 
determination of the turning angle of the residual nucleus in the hard-scattering reaction. This will be a key part of the SRC analysis.

DCH will be replaced by CSC. The reconstruction of hits for the CSC prototype was developed for the first time for the BM@N experiment. The first look at the CSC spatial hits matched with the DCH tracks shows reasonable CSC-DCH correlation. The detectors of the outer tracker (DCH and CSC) provide high hit reconstruction efficiency per layer.

BM@N and SRC teams are on the way to the better understanding of the data for the future obtaining of new physical results.

\section{References}

[1] A.N. Sisakian et al. (NICA Collaboration), J. Phys. G36 (2009) 064069

[2] M. Kapishin, The fixed target experiment for studies of baryonic matter at the Nuclotron (BM@N), Eur. Phys. J. A 52 (2016) 213

[3] R. Weiss, R. Cruz-Torres, N. Barnea, E. Piasetzky, and O. Hen, The Nuclear Contacts and Short-Range Correlations in Nuclei, Phys. Lett. 780 (2018) 211

[4] D.Bederede et al., Nucl. Instrum. Meth. A367 (1995) 88

[5] V. Lenivenko, V. Palichik, Charged particle trajectory reconstruction in multiwire chambers system in the BM@N experiment, JINR preprint P1-2017-26 (2017) 